\title{
Acceleration of dust particles by vortex ring
}

\author{
Z A H I D A E H A N N $1,2,3$, N. L. T S I N T S A D Z E ${ }^{4}$, J. V R A N J E S \\ R. K H A N ${ }^{5}$ and S. POE D T ${ }^{1}$ \\ ${ }^{1}$ Center for Plasma Astrophysics, K. U. Leuven, Celestijnenlaan 200B, \\ 3001 Leuven, Belgium \\ (marsstreet@gmail.com) \\ ${ }^{2}$ National Center for Physics, Quaid-i-Azam University Campus, Islamabad, Pakistan \\ ${ }^{3}$ Blackett Laboratory, Imperial College London, SW72BZ, UK \\ ${ }^{4}$ E. Andronikashvili Institute of Physics, 0171 Georgia \\ ${ }^{5}$ National Tokamak Fusion Program, PO Box 3329, PAEC, Islamabad, Pakistan
}

(Received 11 November 2009, revised 5 May 2010 and accepted 6 May 2010)

\begin{abstract}
It is shown that nonlinear interaction between large amplitude circularly polarized EM wave and dusty plasma leads to a non-stationary ponderomotive force, which in turn produces a vortex ring and magnetic field. Then the ensuing vortex ring in the direction of propagation of the pump wave can accelerate the micron-size dust particles, which are initially at rest and eventually form a non-relativistic dust jet. This effect is purely non-stationary and unlike linear vortices, dust particles do not rotate here. Specifically, it is pointed out that the vortex ring or closed filament can become potential candidate for the acceleration of dust in tokamak plasmas.
\end{abstract}

In the past there have been fairly extensive investigations in the field of nonlinear interaction of high-frequency EM waves or short-pulse laser beams with an electronion plasma [1-18]. Such interactions can produce various types of phenomena, like self-focusing Brilliouin or Raman scattering, filamentation or modulational instability, collisionless shock waves or solitons, wave breaking, absorption of EM waves, generation of vortex rings, quasi-static magnetic fields etc. Spontaneously generated magnetic fields are not only important for laboratory-produced plasmas but also in many cosmic environments, in our Universe as well as in galactic and intergalactic spaces [19-25]. A mechanism for the generation of large quasistatic magnetic fields and vortex ring by a non-potential ponderomotive force resulting from the time-dependent amplitude of EM waves in electron-ion plasma was discussed in Ref. [5]. This way the production of magnetic field can essentially affect the transport phenomena as well as the absorption of an EM field in inertial confinement fusion (ICF) schemes. Indeed, the vortex ring phenomena can have profound influence on laser experimental research [26-28], and at the same time its behavior can be described within the main equations of a continuous medium [13-15]. Most significantly, once this vortex is generated, it develops only under the effect of its own dynamics. Unlike straight vortex filament that is a static phenomenon of plasma, the vortex ring moves relative to the plasma, and at the same time expands noticeably in the process. 
Recently, research on dust in tokamak plasmas has attracted a lot of interest, although existence of these micron-size particles has been known for a long time. Besides some safety threats and engineering issues, the hereness of such heavier particles in fusion devices can influence the plasma operation and performance effectively. Thus, it is expected that dust in plasmas is going to play a requisite role in the next-generation ITER-like fusion devices [29-36].

It has been predicted theoretically that dust particles in tokamak plasma can gain very high velocity and their presence can significantly affect when these collide the tokamak wall, the ejecta far exceed the projectile masses. This not only supplies fresh particles but also releases neutral gas and a runaway effect for wall erosion and plasma contamination that could be a potential hazard for maintaining fusion conditions [33]. Also, if such fast particles exist in the scrape-off layer (SOL), dust impact ionization could be used as a diagnostic. Different mechanisms have been proposed which can cause the acceleration of dust to such high velocities. Among them, ion/dust drag force is mostly believed to play a dominant role, the values of which strongly depend on plasma parameters as well as dust size, shape, temperature and electric charge; it could speed up the dust up to ion flow velocities, which are found to be tens of kilometer per second for toroidal plasma flow near the last closed magnetic surface (LCMS) [33].

de Anglis et al. proposed a mechanism for the acceleration of dust based on stochastic heating [34]. Shukla and Tsintsadze quite recently showed that the normal component of the space charge electric field may accelerate dust particles in the SOL close to the tokamak chamber wall [35].

In this article we propose a mechanism for the acceleration of dust particles by closed filaments or vortex rings that are produced by the action of non-stationary ponderomotive force occurring due to the time dependence of the amplitude of EM wave, which may have effects on the physics of tokamak plasmas.

To consider the propagation of ion cyclotron tranverse EM wave, we follow the standard technique for interaction of high-frequency EM radiation with some accidental and relatively low-frequency plasma perturbations and write down the following equation of motion for dust grains, ions and electrons

$$
\begin{gathered}
\frac{\partial \mathbf{v}_{d}}{\partial t}+\left(\mathbf{v}_{d} \cdot \nabla\right) \mathbf{v}_{d}=-\frac{Z_{d} e}{m_{d}}\left(\mathbf{E}+\frac{1}{c} \mathbf{v}_{d} \times \mathbf{B}\right) \\
m_{\alpha}\left[\frac{\partial \mathbf{v}_{\alpha}}{\partial t}+\left(\mathbf{v}_{\alpha} \cdot \nabla\right) \mathbf{v}_{\alpha}\right]=q_{\alpha}\left(\mathbf{E}+\frac{1}{c} \mathbf{v}_{\alpha} \times \mathbf{B}\right)+\mathbf{F}_{N L}-\frac{1}{n_{\alpha}} \nabla P_{\alpha},
\end{gathered}
$$

where $\alpha$ denotes ions $\left(q_{i}=e\right)$ and electrons $\left(q_{e}=-e\right)$, and $\mathrm{c}$ is the velocity of light. The constant magnetic field is applied in the $z$-direction $B_{0} e_{z}$. The coupling with the intense EM field is described by the coordinate and time-dependent ponderomotive force $[4,6,16,37]$ expressed in terms of the vector potential

$$
\mathbf{F}_{N L}=-m_{\alpha} c^{2}\left[\frac{\omega_{o}}{\omega_{o} \pm \omega_{c \alpha}} \nabla\left(\frac{e^{2}\left|A^{2}\right|}{m_{\alpha}^{2} c^{4}}\right)-\frac{\omega_{c \alpha} \mathbf{k}_{o}}{\left(\omega_{o} \pm \omega_{c \alpha}\right)^{2}} \frac{\partial}{\partial t}\left(\frac{e^{2}\left|A^{2}\right|}{m_{\alpha}^{2} c^{4}}\right)\right],
$$

where $\omega_{c \alpha}=\frac{e B_{o}}{m_{\alpha} c}$ is the cyclotron frequency of the $\alpha$ species, and \pm represents different polarizations. As transverse circularly polarized wave is propagating along magnetic field which is directed along $z$-axis, wave vector $\left(\mathbf{k}_{o}\right)$ has only one component in $z$-direction, i.e. $\left[\mathbf{k}_{o}=\left(0,0, k_{o} \mathbf{e}_{z}\right)\right]$. 
Assuming the condition $n_{o e} / n_{o i} \ll T_{e} / T_{i}$ and for $\omega \sim \omega_{c i}$, we see that the pondermotive force of electrons is much less than that of ions $\left(F_{\text {Pond }}^{e} \ll F_{\text {Pond }}^{i}\right)$, which means that the electric force can be balanced only by the electron pressure gradient (for inertialess electrons, in the momentum equation, the electron pondermotive force is very small in comparison with pressure term). It is to be noted that above pondermotive force expression contains two parts, space dependant and time dependant, where the later is due to magnetic field. In the absence of static magnetic field $\left(B_{0}=0\right)$, the pondermotive force expression reduces to the ordinary one [38]. In an electron-depleted plasma, or equivalently for oscillations well below the electron Debye scale, the ions play a vital role for the excitation of a very low-frequency wave. Hence, the quasi-neutrality condition that we use reads as $\delta n_{i} \simeq Z_{d} \delta n_{d}$. Neglecting the inertial term in (2), and combining the remaining equation with (1) in the limit $v_{d}, v_{i} \ll c$, we get

$\frac{\partial \mathbf{v}_{d}}{\partial t}=-\frac{1}{2 m_{d}} \nabla \frac{v_{d}^{2}}{2}+\mathbf{v}_{\mathbf{d}} \times\left(\nabla \times \mathbf{v}_{d}\right)-\frac{Z_{d} m_{i} c^{2}}{m_{d}}\left[\frac{\omega_{o}}{\omega_{o}-\omega_{c i}} \nabla \Psi^{2}-\frac{\omega_{c i} \mathbf{k}_{o}}{\left(\omega_{o}-\omega_{c i}\right)^{2}} \frac{\partial \Psi^{2}}{\partial t}\right]-\frac{Z_{d}}{n_{i} m_{d}} \nabla P_{i}$,

where $\Psi=e A /\left(m_{i} c^{2}\right)$ is a dimensionless vector potential. Taking the curl of both sides, we obtain

$$
\frac{\partial \boldsymbol{\Omega}}{\partial t}=\nabla \times \mathbf{v}_{d} \times \boldsymbol{\Omega}+\frac{Z_{d} \omega_{c i} m_{i} c^{2}}{m_{d}\left(\omega_{o}-\omega_{c i}\right)^{2}} \nabla \frac{\partial \Psi^{2}}{\partial t} \times \mathbf{k}_{o} .
$$

Here $\boldsymbol{\Omega}=\nabla \times \mathbf{v}_{d}$ represents the vorticity of the dust velocity. Considering $l>v_{d} t$, where $l$ and $t$ represent the characteristic spatial and time scale lengths, respectively, will allow us to neglect the first term on the right-hand side in comparison to the term on the left-hand side in (4). As a result, we obtain a simple relation between the vorticity and the source,

$$
\boldsymbol{\Omega}=\frac{Z_{d} \omega_{c i} m_{i} c^{2}}{m_{d}\left(\omega_{o}-\omega_{c i}\right)^{2}} \nabla \Psi^{2} \times \mathbf{k}_{o} .
$$

For a circularly polarized EM wave propagating along the $z$-axis, the vorticity has only two components, $\boldsymbol{\Omega}_{x}$ and $\boldsymbol{\Omega}_{y}$. Thus, vortices are produced in the plane perpendicular to the propagation of the pump wave, i.e. we have the formation of vortex rings or close filaments.

Now we investigate (4) in the cylindrical coordinates $r, \theta, z$ at the center of the ring, which clearly shows that the vorticity has only one component, $\boldsymbol{\Omega}=\boldsymbol{\Omega}_{\theta} \mathbf{e}_{\theta}$, and that the particle velocity is $\mathbf{v}_{d}=v_{r} \cdot \mathbf{e}_{r}+v_{z} \cdot \mathbf{e}_{z}$, where $\left(\mathbf{e}_{r}, \mathbf{e}_{\theta}, \mathbf{e}_{z}\right)$ are the unit vectors. Expressing $(\boldsymbol{\Omega} \cdot \nabla)=\left(\Omega_{\theta} / r\right)(\partial / \partial \theta), \partial v / \partial \theta=v_{r} \mathbf{e}_{\theta}$, and $v_{r}=d r / d t$ in (4), we obtain

$$
\frac{d}{d t}\left(\frac{\Omega_{\theta}}{n_{d} r}\right)=\frac{Z_{d} \omega_{c i} m_{i} c^{2}}{m_{d}\left(\omega_{o}-\omega_{c i}\right)^{2}} \frac{k_{o}}{n_{d} r} \frac{\partial}{\partial r}\left(\frac{\partial \Psi^{2}}{\partial t}\right) .
$$

From (3)-(6) it can be noticed that the time dependence of the pondermotive force does not conserve the velocity circulation, $\Gamma_{d}=\oint \mathbf{v}_{d} \mathbf{d r}=\int_{s} \int \boldsymbol{\Omega} \mathbf{d s}$. The same follows for frozen-in condition [39] which does not take place but only for the wake vorticity $\left(\partial \Psi^{2} / \partial t=0\right)$

$$
\frac{\Omega_{\theta}}{n_{d} r}=\text { const. }
$$


Equations (4)-(6) indicate that if at the initial instant of time there are no vortices of the dust fluid flow at a given point, they will be generated by the circularly polarized EM field. It is important to note that if there are $N$ point-like vortices (filaments), then these filaments can interact with each other, leading eventually to the merging of vortices, to the decaying of a vortex into two other vortices, to the annihilation of vortices etc. Another interesting feature is that here unlike linear vortices, dust particles do not rotate.

We have shown above how a circularly polarized EM wave generates closed filaments, values of which can be defined by the intensity of the pump. We now demonstrate that there is a mechanism of the acceleration of the dust particles up to a particular velocity due to the vortex ring. For this, considering the inverse problem, i.e. by a given vortex, we can define the velocity of the dust grains $\left(\mathbf{v}_{d}\right)$ at any point in the plasma. To this end, we assume that the plasma is at rest until the vortex is generated, $\boldsymbol{\Omega}=\nabla \times \mathbf{v}_{\mathbf{d}}$. Let us introduce the vector $\mathbf{P}$ such that $\nabla \cdot \mathbf{P}=0$, and $\mathbf{v}_{\mathbf{d}}=\nabla \times \mathbf{P}$. For $\mathbf{P}$ we can write down the following equation:

$$
\nabla^{2} \mathbf{P}=-\boldsymbol{\Omega}
$$

which has solution of the form

$$
\mathbf{P}=\frac{1}{4 \pi} \int \frac{\boldsymbol{\Omega}\left(\mathbf{r}^{\prime}\right) d \mathbf{r}^{\prime}}{R},
$$

where $d \mathbf{r}^{\prime}=d x^{\prime} d y^{\prime} d z^{\prime}, R=\left[\left(x-x^{\prime}\right)^{2}+\left(y-y^{\prime}\right)^{2}+\left(\xi-z^{\prime}\right)^{2}\right]^{1 / 2}, \xi=z-u_{g} t$ and $u_{g}=\frac{2 k_{c} c^{2} \omega_{c d}}{\omega_{p d}^{2}}$ is the group velocity of the pump wave.

By knowing the vector $\mathbf{P}$, we can define the velocity $\mathbf{v}_{d}$ at any point in the plasma

$$
\mathbf{v}_{d}(\mathbf{r}, t)=\nabla \times \frac{1}{4 \pi} \int \frac{\Omega\left(\mathbf{r}^{\prime}\right) d \mathbf{r}^{\prime}}{R} .
$$

Let us now consider a vortex ring with radius $\rho_{o}$, and characterize the position of the variable on the vortex ring by the angle $\alpha$. Any point on the vortex filament in the Cartesian coordinates is determined by $x^{\prime}=\rho_{o} \cos \alpha, y^{\prime}=\rho_{o} \sin \alpha$ and $z^{\prime}=0$.

In order to calculate the integral in (9), we assume that $\Psi^{2}=\Psi_{0}^{2} u_{g} \tau \delta\left(z^{\prime}-\right.$ $\left.u_{g} \tau\right) \theta\left(r^{\prime}-\rho_{o}\right)$, and $\mathbf{P}$ has only one component, $P_{\theta}=P_{\theta}(\mathbf{r}, z)$ [39]. From (9), we have

$$
P_{\theta}(r, \xi)=\frac{\omega_{c i} m_{i} c^{2} k_{o} u_{g} \tau \rho_{o} \Psi^{2}}{m_{d} \pi\left(\omega_{o}-\omega_{c i}\right)^{2}} \oint \frac{d \alpha \cdot \cos \alpha}{R},
$$

here $R=\left(r^{2}+\xi^{2}+\rho_{o}^{2}-2 r \rho_{o} \cos \alpha\right)^{1 / 2}, \tau$ is the duration of EM field and the integral is

$$
\oint \frac{\cos \alpha d \alpha}{R}=\frac{4}{\eta} \sqrt{\frac{1}{r \rho_{o}}}\left[\left(1-\frac{\eta^{2}}{2}\right) K(\eta)-E(\eta)\right],
$$

where $\eta^{2}=4 r \rho_{o} /\left[\left(r+\rho_{o}\right)^{2}+\zeta^{2}\right], K$ and $E$ are the elliptical integrals of the first and second kind,

$$
\begin{gathered}
K(\eta)=\int_{0}^{2 \pi} \frac{d \beta}{\sqrt{1-\eta^{2} \sin ^{2} \beta}} \\
E(\eta)=\int_{0}^{2 \pi} \sqrt{1-\eta^{2} \sin ^{2} \beta} d \beta
\end{gathered}
$$


and $\beta=(\alpha-\pi) / 2$. As a result we have

$$
P_{\theta}(r, \xi)=\frac{\omega_{c i} m_{i} c^{2} k_{o} u_{g} \tau \Psi_{0}^{2}}{m_{d}\left(\omega_{o}-\omega_{c i}\right)^{2} \eta} \times \sqrt{\frac{\rho_{o}}{r}}\left[\left(1-\frac{\eta^{2}}{2}\right) K(\eta)-E(\eta)\right] .
$$

The components of the velocity can be written as

$$
v_{d r}=-\frac{\partial P_{\theta}}{\partial z}, \quad v_{d z}=\frac{1}{r} \frac{\partial}{\partial r}\left(r P_{\theta}\right)
$$

or

$$
\begin{aligned}
& v_{d r}=\frac{\omega_{c i} m_{i} c^{2} k_{o} u_{g} \tau \Psi_{0}^{2}}{m_{d}\left(\omega_{o}-\omega_{c i}\right)^{2} 2 \pi r \sqrt{\left(r+\rho_{o}\right)^{2}+\zeta^{2}}} \times \zeta\left[-K(\eta)+\frac{r^{2}+\rho_{o}^{2}+\zeta^{2}}{\left(\rho_{o}-r\right)^{2}+\zeta^{2}} E(\eta)\right], \\
& v_{d z}=\frac{\omega_{c i} m_{i} c^{2} k_{o} u_{g} \tau \Psi_{0}^{2}}{m_{d}\left(\omega_{o}-\omega_{c i}\right)^{2} 2 \pi \sqrt{\left(r+\rho_{o}\right)^{2}+\zeta^{2}}} \times\left[K(\eta)+\frac{r^{2}+\rho_{o}^{2}+\zeta^{2}}{\left(\rho_{o}-r\right)^{2}+\zeta^{2}} E(\eta)\right] .
\end{aligned}
$$

Now we consider two cases, for the first, assuming $r \rightarrow 0$, i.e. on the axis

$$
v_{d r}=0, \quad v_{d z}=\frac{m_{i} c^{2} \omega_{c i} k_{o} u_{g} \tau \Psi_{0}^{2}}{m_{d}\left(\omega_{o}-\omega_{c i}\right)^{2}\left(\rho_{o}^{2}+\zeta^{2}\right)^{1 / 2}} .
$$

Recalling that $\xi-\zeta-u_{g} t=0$, and for the maximum, we write

$$
v_{d z, \max }=\frac{m_{i} c^{2} \omega_{c i} k_{o} u_{g} \tau \Psi_{0}^{2}}{m_{d} \rho_{o}\left(\omega_{o}-\omega_{c i}\right)^{2}} .
$$

Now we consider the case at the point near the filament, i.e. $r=\rho_{o}$ and $\xi=\zeta-u_{g} \tau=$ 0 . In this case $\eta^{2}=1, E=\pi / 2$, but $K(1)$ is logarithmically divergent at the lower limit as

$$
K=\frac{1}{2} \int_{0}^{\pi} \frac{d \alpha}{\sin \alpha / 2} \simeq \int_{0}^{\pi} \frac{d \alpha}{\alpha} .
$$

In reality, the closed filament as a ring has a finite size denoted by $r_{o}\left(r_{o}\right.$ is the core radius of the thin vortex ring), so there must be a cut off at a value $\alpha \sim r_{o} / \rho_{o}$, and $K(1)=\ln \rho_{o} / r_{o}$. The $z$-component of the velocity now becomes

$$
v_{d z}=\frac{m_{i} c^{2} \omega_{c i} k_{o} u_{g} \tau \Psi_{0}^{2}}{m_{d} \rho_{o}\left(\omega_{o}-\omega_{c i}\right)^{2}} \ln \frac{\rho_{o}}{r_{o}} .
$$

Using the simple relation between vorticity and source we have obtained expressions of dust speed [Equations (12) and (13)] which particles gain from the filaments and have shown vortex ring can generate a collimated dust jet along the propagation of EM wave. We take some typical parameters that are representative of Tokamak plasmas: $n_{d}=3 \times 10^{13} \mathrm{~cm}^{-3}$, dust mass density $=2 \mathrm{~g} \mathrm{~cm}^{-3}, n_{i}=10^{14} \mathrm{~cm}^{-3}, \tau=10^{-4} \mathrm{~s}$, radius of vortex ring $\rho_{o}=10 \mathrm{~cm}$ (it could be taken as $5 \mathrm{~cm}$ ), wavelength $\lambda=1 \mathrm{~cm}$, intensity of waves $I=10^{7}$ watt $\mathrm{cm}^{-2}$ and external magnetic field $B_{o}=10^{5} \mathrm{G}$. For deuterium plasma case $\Psi_{0}^{2} \sim 10^{-6}$. Hence, the dust velocity, estimated from (18), turns out to be of the order of $10^{2} \mathrm{~cm} \mathrm{~s}^{-1}$. We emphasize that above consideration can be applied to the acceleration of dust particles in the SOL of the Tokamak.

Now we manifest how the non-stationary pondermotive force of the EM wave, which creates slowly varying electric fields and vector potentials, can generate magnetic fields. 
A simple expression for this can be obtained by taking the curl of the momentum equation for inertialess ions

$$
\nabla \times \mathbf{E}=-\frac{1}{e} \nabla \times \mathbf{F}_{N L}
$$

Using the Faraday law $\nabla \times \mathbf{E}=-(\partial \mathbf{B} / \partial t) / c,(21)$ becomes

$$
\frac{\partial \mathbf{B}}{\partial t}=\frac{c}{e} \nabla \times \mathbf{F}_{N L}=\frac{c}{e}\left[\frac{\omega_{c i} m_{i} c^{2}}{\left(\omega_{o}-\omega_{c i}\right)^{2}} \nabla \frac{\partial \Psi^{2}}{\partial t} \times \mathbf{k}_{o}\right] .
$$

Integrating both sides we get

$$
\mathbf{B}=\frac{\omega_{c i} m_{i} c^{3}}{e\left(\omega_{o}-\omega_{c i}\right)^{2}} \nabla \Psi^{2} \times \mathbf{k}_{o} .
$$

The $x$ and $y$ components of the above equation are

$$
\begin{gathered}
\frac{e B_{x}}{m_{i} c}=\frac{c^{2} \omega_{c i} \mathbf{k}_{o}}{\left(\omega_{o}-\omega_{c i}\right)^{2}} \frac{\partial \Psi^{2}}{\partial y}, \\
\frac{e B_{y}}{m_{i} c}=-\frac{c^{2} \omega_{c i} \mathbf{k}_{o}}{\left(\omega_{o}-\omega_{c i}\right)^{2}} \frac{\partial \Psi^{2}}{\partial x} .
\end{gathered}
$$

Squaring and then addition of (24) and (25) give us generation of magnetic field

$$
\frac{e B}{m_{i} c}= \pm \frac{k_{o} \omega_{c i} c^{2}}{\left(\omega_{o}-\omega_{c i}\right)^{2}} \frac{\partial \Psi^{2}}{\partial r}, \quad r=\left(x^{2}+y^{2}\right)^{1 / 2} .
$$

Equations (24)-(26) show that magnetic field is generated in the plane perpendicular to the starting magnetic field $B_{o} \mathbf{e}_{z}$, and the components of the generated magnetic field are determined by both, the parameters of the plasma and the intensity of the pump wave. Using parameters given above, magnetic field strength, estimated from (23), turns out to be $\sim 4 \times 10^{5} \mathrm{G}$. Here we specify that this spontaneously generated magnetic field can influence the energy transport and propagation of EM waves in Tokamak fusion plasmas.

To summarize, the main idea here is to identify a new mechanism for the generation of vortex ring which can accelerate dust particles to very high velocity resulting in the formation of a non-relativistic dust jet by employing the nonlinear interaction of circularly polarized EM wave with dusty plasma. Specifically, this interaction leads to a non-stationary pondermotive force which pushes the ions locally and creates slowly varying electric fields and vector potentials. The latter, in turn, generates vortex ring and quasi-stationary magnetic fields in the perpendicular direction. Then we considered an interesting inverse problem that the ensuing vortex ring becomes a means for the formation of dust jets (dust particles were initially in an equilibrium state) which are emitted after gaining acceleration. By some simple estimates, we assessed the relevance of the above analysis for tokamak plasmas and found that new mechanism of accelerating dust particles by propagation of ion cyclotron EM wave can help in removing dust particles from next-generation Tokamak like fusion devices. In addition, generation of magnetic field is a ubiquitous phenomenon relevant to astrophysics and this can also affect energy transport in ICF schemes. 


\section{Acknowledgements}

The results presented here are obtained in the framework of Geconcerteerde Onderzoeksacties 'BOF' and 'LRD' (K.U. Leuven), Georgian Science Foundation Grant Project No. 1-4/16(GNSF/ST09_305_4-140 and Higher Education Commission of Pakistan.

\section{References}

[1] Akhiezer, I. A., Daneliya, L. D. and Tsintsadze, N. L. 1964 Sov. Phys. JETP 19, 208.

[2] Zakharov, V. 1972 Sov. Phys. JETP 35, 908.

[3] Tsintsadze, N. L. 1974 Phys. Lett. 50A, 33.

[4] Karpman, V. I. and Washimi, H. 1976 Sov. Phys. JETP 44, 528.

[5] Berezhiani, V. I., Tsintsadze, N. L. and Tskhakaya, D. D. 1980 J. Plasma. Phys. 24, 15.

[6] Tsintasdze, N. L. and Watanabe, M. 1980 Sov. J. Plasma Phys. 6, 6.

[7] Tsintsadze, N. L., Mima, K., Tsintsadze, L. N. and Nishikawa, K. 2002 Phys. Plasmas. 9, 4270.

[8] Freund, H. P., Liu, C. S. and Kulsrud, R. M. 1981 J. Plasma Phys. 25, 465.

[9] Stamper, J. A. 1992 Las. Part. Beams 9, 841.

[10] Tsintsadze, L. N., Callebaut, D. K. and Tsintsadze, N. L. 1996 J. Plasma Phys. 55, 407.

[11] Sudan, R. N. 1993 Phys. Rev. Lett. 70, 3075; Askaryan, G. A., Bulanov, S. V., Pegoraro, F. and Pukhov, A. M. 1994 Sov. Phys. JETP 60, 251; Gorbunov, L. M., Mora, P. and Antonsen, T. M. 1996 Phys. Rev. Lett. 76, 2945.

[12] Borghesi, M., MacKinnon, A. J., Bell, A. R., Gaillard, R. and Willi, O. 1998 Phys. Rev. Lett. 81, 112.

[13] Wilks, S. C., Kruer, W. L., Tabak, M. and Langdon, A. B. 1992 Phys. Rev. Lett. 69, 1383.

[14] Tsintsadze, L. N., Mima, K. and Nishikawa, K. 1998 Plasma Phys. Controlled Fusion 40, 1933.

[15] Tsintsadze, L. N., Nishikawa, K., Tajima, T. and Mendonca, J. T. 1999 Phys. Rev. E 60, 7435.

[16] Tsintsadze, N. L., Pajouh, H. H., Tsintsadze, L. N., Mendonca, J. T. and Shukla, P. K. 2000 Phys. Plasmas 7, 2348.

[17] Vranjes, J., Saleem, H. and Poedts, S. 2007 Phys. Plasmas 14, 034504.

[18] Nakamura, T. and Mima, K. 2008 Phys. Rev. Lett. 100, 205006.

[19] Tatarakis, M., Watts, I., Beg, F. N., Clark, E. L., Dangor, A. E., Gopal, A., Haines, M. G., Norreys, P. A., Wagner, U., Wei, M. S., Zepf, M. and Krushelnick, K. 2002 Nature (London) 415, 280; Wagner, U., Tatarakis, M., Gopal, A., Beg, F. N., Clark, E. L., Dangor, A. E., Evans, R. G., Haines, M. G., Mangles, S. P. D., Norreys, P. A., Wei, M. S., Zepf, M. and Krushelnick, K. 2004 Phys. Rev. E 70, 026401.

[20] Kolb, E. W. and Turner, M. S. 1994 The Early Universe. Reading, MA: Addison-Wesley.

[21] Ryu, D., Kang, H., Cho, J. and Das, H. 2008 Science 320, 909.

[22] Widrow, L. M. 2002 Rev. Mod. Phys. 74, 775.

[23] Kulsrud, R. M. and Zweibel, E. G. 2008 Rep. Prog. Phys. 71, 046901.

[24] Bernet, M. L., Miniati, F., Lilly, S. J., Kronberg, P. P. and Dessauges-Zavadsky, M. 2008 Nature (London) 454, 302.

[25] Bobin, J. L. 1983 Phys. Rep. 122, 173.

[26] Hatchett, S. P., Brown, C. G., Cowan, T. E., Henry, E. A., Johnson, J. S., Key, M. H., Jeffrey, Koch, A., Langdon, A. B., Lasinski, B. F., Lee, R. W., Mackinnon, A. J., Pennington, D. M., Perry, M. D., Phillips, T. W., Sangster, M. C., Singh, M. S., Snavely, R. A., Stoyer, M. A., Wilks, S. C. and Yasuike, K. 2000 Phys. Plasmas 7, 2076. 
[27] Snavely, R. A., Key, M. H., Hatchett, S. P., Cowan, T. E., Roth, M., Phillips, T. W., Stoyer, M. A., Henry, E. A., Sangster, T. C., Singh, M. S., Wilks, S. C., MacKinnon, A., Offenberger, A., Pennington, D. M., Yasuike, K., Langdon, A. B., Lasinski, B. F., Johnson, J., Perry, M. D. and Campbell, E. M. 2000 Phys. Rev. Lett. 85, 2945.

[28] Wilks, S. C., Kruer, W. L., Tabak, M. and Langdon, A. B. 1992 Phys. Rev. Lett. 69, 1383.

[29] Narihara, K. 2001 Nucl. Fusion 411967.

[30] Rudakov, D. L., West, W. P., Wong, C. P. C., Brooks, N. H., Evans, T. E., Fenstermacher, M. E., Groth, M., Krasheninnikov, S. I., Lasnier, C. J., McLean, A. G., Pigarov, A. Y., Solomon, W. M., Antar, G. Y., Boedo, J. A., Doerner, R. P., Hollmann, E. M., Hyatt, A. W., Moyer, R. A. and Watkins, J. G. 2007 J. Nucl. Matter. 227, 363.

[31] Castaldo, C., Ratynskaia, S., Pericoli1, V., de Angelis, U., Rypdal, K., Pieroni, L., Giovannozzi1, E., Maddaluno, G., Marmolino, C., Rufoloni, A., Tuccillo, A., Kretschmer, M. and Morfill, G. E. 2007 Nucl. Fusion 47, L5-L9.

[32] Ratynskaia, S. 2008 Nucl. Fusion 48, 015006.

[33] de Angelis, U., Marmolino, C. and Tsytovich, V. 2005 Phys. Rev. Lett. 95, 095003.

[34] de Angelis, U., Ivlev, A., Tsytovich, V. and Morfill, G. 2005 Phys. Plasmas 12, 052301.

[35] Shukla, P. K. and Tsintsadze, N. L. 2008 Phys. Lett. A 372, 2053.

[36] Ehsan, Z., Tsintsadze, N. L., Murtaza, G. and Shah, H. A. 2009 Phys. Plasmas 16, 023702.

[37] Ehsan, Z., Tsintsadze, N. L., Vranjes, J. and Poedts, S. 2009 Phys. Plasmas 16, 053702.

[38] Tsintsadze, N. L., Ehsan, Z., Shah, H. A. and Murtaza, G. 2006 Phys. Plasmas 13, 072103; 2006 In: Proceedings of the Second International Symposium on Unconventional Plasmas (ed. D. K. Callebaut). Eindhoven: Netherlands, Eindhoven, 2006, vol. 161.

[39] Saffman, P. G. 1995 Vortex Dynamics. Cambridge: Cambridge University Press. 